\title{
Anti-CHMP5 single chain variable fragment antibody retrovirus infection induces programmed cell death of AML leukemic cells in vitro
}

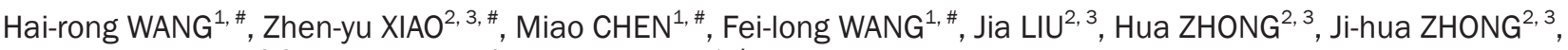 \\ Ren-rong OU-YANG ${ }^{2,3}$, Yan-lin SHEN ${ }^{4}$, Shu-ming PAN ${ }^{1, *}$ \\ ${ }^{1}$ Emergency Department, Xin-Hua Hospital, Shanghai Jiao Tong University School of Medicine, Shanghai 200092, China; ${ }^{2}$ Department \\ of Hematology, Ren-Ji Hospital, Shanghai Jiao Tong University School of Medicine, Shanghai 200127, China; ${ }^{3}$ Shanghai Institute of \\ Hematology, Shanghai 200025, China; ${ }^{4}$ Department of Pharmacy, Xin-Hua Hospital, Shanghai Jiao Tong University School of Medicine, \\ Shanghai 200092, China
}

Aim: Over-expressed CHMP5 was found to act as oncogene that probably participated in leukemogenesis. In this study, we constructed the CHMP5 single chain variable fragment antibody (CHMP5-scFv) retrovirus and studied the changes of programmed cell death (PCD) of AML leukemic cells after infection by the retrovirus.

Methods: The anti-CHMP5 KC14 hybridoma cell line was constructed to generate monoclonal antibody of CHMP5. The protein expression of CHMP5 was studied using immunofluorescence analysis. pMIG-CHMP5 scFv antibody expressible retroviral vector was constructed to prepare CHMP5-ScFv retrovirus. AML leukemic U937 cells were infected with the retrovirus, and programmed cell death was studied using confocal microscope, FCM and Western blot.

Results: We obtained a monoclonal antibody of CHMP5, and found the expression of CHMP5 was up-regulated in the leukemic cells. After U937 cells were infected with CHMP5-scFv retrovirus, CHMP5 protein was neutralized. Moreover, the infection resulted in a significant increase in apoptosis and necrosis of U937 cells. In U937 cells infected with CHMP5-scFv retrovirus, apoptosis-inducing factor (AIF)-mediated caspase-independent necrotic PCD was activated, but autophagic programmed cell death was not observed. Neither the intrinsic nor extrinsic apoptotic PCD pathway was activated. The granzyme B/perforin-mediated caspase-dependent apoptotic PCD pathway was not activated.

Conclusion: CHMP5-ScFv retrovirus can neutralize the abnormally high levels of the CHMP5 protein in the cytosol of AML leukemic U937 cells, thereby inducing the programmed cell death of the leukemic cells via AIF-mediated caspase-independent necrosis and apoptosis.

Keywords: leukemia; CHMP5; monoclonal antibody; single chain variable fragment antibody (scFv); retrovirus; programmed cell death; apoptosis; necrosis; autophagy; apoptosis-inducing factor (AIF)

Acta Pharmacologica Sinica (2012) 33: 809-816; doi: 10.1038/aps.2012.38; published online 21 May 2012

\section{Introduction}

In adults, acute myeloid leukemia (AML) is by far the most common type of acute leukemia. It is a cancer of the bone marrow and is characterized by a mutation in a hematopoietic stem or progenitor cell, which develops into the accumulation of highly proliferative dysfunctional and immature myeloid cells. These abnormal cells eventually dominate hematopoietic niches, such as the bone marrow, and result in abnormal

\footnotetext{
\# These authors contributed equally to this work.

* To whom correspondence should be addressed.

E-mail panshuming@live.com

Received 2012-02-09 Accepted 2012-03-23
}

peripheral blood counts due to high number of myeloblasts. Over the past several decades, improvements in chemotherapeutic regimens and supportive care have resulted in significant but modest progress in treating AML, but AML remains a fatal disease for most patients ${ }^{[1]}$. A better molecular definition and the elucidation of the physiopathology of AML combined with the development of new and targeted therapies may result in a better prognosis for patients with AML in the future.

The human charged multivesicular body protein 5 (CHMP5) is a member of the CHMP family of coiled-coil proteins ${ }^{[2]}$. The multivesicular body (MVB) is a particular type of late endosomes, which are crucial intermediates in the internalization 
of nutrients, ligands and receptors through the endolysosomal system. It plays a crucial role in sorting membrane proteins that are destined for degradation or routing to the lysosome $\mathrm{e}^{[3,4]}$. These proteins, such as activated growth-factor, hormone and cytokine receptors, are internalized into vesicles forming an MVB, the contents of the MVB are then transferred to lysosomes for degradation ${ }^{[2,5,6]}$. CHMP5 is responsible for the final conversion of late endosomal MVB to lysosomes and plays a critical role in the downregulation of signaling pathways through receptor degradation ${ }^{[2,7]}$.

Functional analyses of CHMP5 are performed using an RNAi and protein overexpression strategy in our lab. We found CHMP5 is an anti-apoptotic gene ${ }^{[8]}$, which is consistent with previous findings by Shahmoradgoli et al ${ }^{[9]}$. Moreover, our data confirmed previous findings showing that CHMP5 is significantly increased in de novo or relapsed acute myeloid leukemia, indicating that it may participate in leukemogenesis ${ }^{[8,9]}$. Immunofluorescence experiments using a monoclonal antibody produced by our lab suggested that the CHMP5 protein was expressed at low levels in the cytosol of $293 \mathrm{~T}$ cells and normal blood mononuclear cells from healthy volunteers. Interestingly, the CHMP5 protein was highly expressed in the cytosol of AML leukemic cells (unpublished data). These findings indicate that highly expressed CHMP5 protein may participate in leukemogenesis, and the abnormal expression of the CHMP5 protein may be a target for gene therapy.

Recently, recombinant antibodies targeting tumor-associated antigens (TAAs) or tumor-specific antigens (TSAs), gene therapy and immunomodulators have transformed the traditional chemotherapy based only on anticancer drugs. TAAs or TSAs are proteins or other molecular species that are expressed in a specific tumor type that can be targeted for diagnostic and immunotherapy purposes ${ }^{[10]}$. Tumor-specific recombinant antibodies have been employed in diagnosis and therapy, such as the anti-CD20 monoclonal antibody (McAb), either as single agents or in combination with chemotherapy. These agents have resulted in large improvements in non-Hodgkin's lymphoma survival rates. The main pitfalls of utilizing antibodies to tumor-specific antigens in cancer diagnosis and therapy are the immune response to non-human antibodies and the large size of antibodies, which hinders their access to the large tumors or into cells ${ }^{[10]}$.

The first single chain variable fragment antibody $\left(\mathrm{scFv}_{\mathrm{V}}\right)$ was developed in 1988. An scFv is an antibody composed of heavy- and light-chain variable regions that are joined by an interchain polypeptide linker. The $\mathrm{scFv}$ is one of the smallest fragments to be produced that shows equivalent binding affinity to the parent Fab (fragment antigen-binding) fragment ${ }^{[1,12]}$. $\mathrm{ScFv}$ can be conjugated to different types of molecules, such as radioisotopes and toxins, or expressed as an intracellular antibody (intrabody) in a specific intracellular compartment, where it can interfere with the function of the targeted antigen at the level of a specific domain ${ }^{[13]}$. Human single chain antibody fragments offer the advantage of being expressed as a single polypeptide, and their small size means that they can serve as a highly safe, selective and effective diagnostic and therapeutical tool ${ }^{[10,14-20]}$. The $\mathrm{scFv}$ on target molecules within cells or intrabodies provides a useful tool for research as well as for control of diseases such as human immunodeficiency virus type 1 infection and other diseases ${ }^{[13,15,16,21]}$.

As we mentioned previously, the expression of the antiapoptotic protein CHMP5 was dramatically up-regulated in the cytosol of AML leukemic cells, which was distinct from the expression in normal mononuclear cells. Infecting AML leukemic cells with an anti-CHMP5 scFv retrovirus may induce programmed cell death by neutralizing the excess antiapoptotic CHMP5 protein.

\section{Materials and methods}

\section{Cell culture and transfection}

Human HEK293T (human embryonic kidney) cells and U937 cells were obtained from the Shanghai Institute of Hematology (Shanghai, China). The anti-CHMP5 KC14 hybridoma cell line was constructed by our lab. The cells were cultured in RPMI1640 supplemented with $10 \%(v / v)$ fetal bovine serum (FBS), $100 \mathrm{U} / \mathrm{mL}$ penicillin and $100 \mu \mathrm{g} / \mathrm{mL}$ streptomycin at $37^{\circ} \mathrm{C}$ and $5 \%(v / v) \mathrm{CO}_{2}$. The media for culturing the hybridoma cell line KC14 contained 1×HAT (Hypoxathine, Aminopterin and Thymidine).

Generation of the anti-CHMP5 hybridoma cell line and preparation of the anti-CHMP5 monoclonal antibody

Antheprot 4.3 was used to select three antigenic epitopes of the CHMP5 protein. These antigenic epitopes were synthesized and used to generate the CHMP5 polyclonal antibody. Western blot analysis revealed that the polyclonal antibody derived from the KC14 antigenic epitope performed best. The amino acid sequence of KC14 was N'-KAK PKA PPP SLT DC-C'. The hybridoma was constructed according to Ball et al ${ }^{[22]}$. Briefly, we conjugated the KC14 peptide to bovine serum albumin (BSA). BALB/c mice were immunized against the synthetic peptide of the CHMP5 protein. The spleen cells of the mice were fused with Sp2/0 mouse myeloma cells. The titers of the monoclonal antibody $(\mathrm{McAb})$ were tested by ELISA.

\section{Cloning of the $\mathrm{VH}$ and $\mathrm{VL}$ regions from the KC14 hybridoma}

Total RNA extracted from the KC14 hybridoma was converted into cDNA using reverse transcriptase. PCR was performed with mouse primers for VL and VH. The primers were designed according to references ${ }^{[23,24]}$. The VL sense primer was: 5'-CCT CTA GAG ACA TTG TG-3'; the VL antisense primer was: 5'-GCC CTT GGC TCG AGT TTT-3'; the VH sense primer was: 5'-ATG AGT CCT GAA CTA ACC TTG AAT C-3'; and the VH antisense primer was: 5'-TCA TTC TAG CCC TTT CCC TGT AGC C-3'. PCR products were purified, TOPO-TA was cloned into the T-vector, and the recombinant vector was sequenced to obtain DNA sequence of VL and VH.

pBabe-CHMP5 scFv antibody retroviral expression vector construction

The pBabe-puro retroviral vector was obtained from the 
Shanghai Institute of Hematology (Shanghai, China). We first inserted the VL sequences into pBabe to generate the pBabe$\mathrm{VL}$ recombinant vector. The VL sense primer included restriction sites for the endonuclease $B a m \mathrm{H} \mathrm{I}$, and the antisense primer included restriction sites for the endonuclease EcoR I. PCR was performed using cDNA of the KC14 hybridoma cells to obtain VL sequences. The purified PCR products and the $\mathrm{pBabe}$-Puro retroviral vector were digested with restriction enzymes BamH I and EcoR I, respectively. The DNA Ligation Kit Ver 2.0 (Takara, Tokyo, Japan) was used to ligate the VL sequences with pBabe to produce the pBabe-VL recombinant vector. Subsequently, we chemically synthesized a linker oligonucleotide. The sense sequence of linker was 5'-AAT TCG GTG GTG GTG GAT CCG GTG GTG GTG GTT CTG GCG GCG GCG GCT CCG-3' and the antisense sequence of linker was 5'-TCG ACG GAG CCG CCG CCG CCA GAA CCA CCA CCA CCG GAT CCA CCA CCA CCG-3'. The underlined portion shows the EcoR I site. After annealing with its counterpart oligonucleotide, the EcoR I sticky end may form at the $5^{\prime}$ terminal end of the linker dsDNA. The Sal I site is shown in italics. After annealing with its counterpart oligonucleotide, the Sal I sticky end may form at the 3' terminal end of the linker dsDNA. After the pBabe-VL recombinant vector was cut with the EcoR I and Sal I restriction enzymes, the linker dsDNA was introduced to form the pBabe-VL-linker recombinant vector. Subsequently, both $\mathrm{VH}$ sense and antisense primers were added with restriction sites for the endonuclease Sal I and VH was introduced into pBabe-VL-linker to form the pBabe-CHMP5 scFv antibody retroviral expression vector.

pMIG-CHMP5 scFv antibody retroviral expression vector construction

The pMIG-GFP retroviral vector, gag/pol and VSV-G were obtained from the Shanghai Institute of Hematology (Shanghai, China). Because the pBabe-CHMP5 scFv antibody retroviral expression vector did not contain the GFP (green fluorescence protein) sequence, it was difficult for us to evaluate the infection efficiency after transient transfection. Therefore, we utilized the pMIG-GFP retroviral vector to construct pMIGCHMP5 scFv antibody retroviral expression vector. The primer pairs $5^{\prime}$-tac gtt aga tct ATG ACC CAG TCT CCT GCT$3^{\prime}$ and $5^{\prime}$-tag att gtt aac GCA TTC TAG CCC TTT CCC-3' were used, and the pBabe-CHMP5 scFv antibody retroviral expression vector served as the DNA template for PCR to obtain the CHMP5 ScFv sequence. The purified PCR products and the pMIG-GFP retroviral vector were digested with the $B g l \mathrm{I}$ and Hpa I restriction enzymes, respectively. The DNA Ligation Kit Ver 2.0 was used to ligate the CHMP5 scFv sequence with pMIG to generate the pMIG-CHMP5 scFv antibody retroviral expression vector.

\section{pMIG-CHMP5 scFv retrovirus preparation and infection}

To prepare the virus, $293 \mathrm{~T}$ cells were cotransfected with the empty pMIG vector or the pMIG-CHMP5 scFv antibody retroviral expression plasmid and gag/pol and VSV-G using the FuGENE 6 transfection reagent (Roche, USA) ${ }^{[25,26]}$. One day after transfection, the medium was changed, and collection of the virus-containing medium began on $\mathrm{d} 2$. The medium was filtered through a $0.22-\mu \mathrm{m}$ filter. U937 cells were then infected with the retroviral supernatants. The clones expressing the desired genes were sorted by a flowcytometer (FCM), and the GFP-positive cells were selected ${ }^{[26]}$.

\section{Immunofluorescence analysis}

Our CHMP5 McAb was used to detect the CHMP5 protein in U937 cells by immunofluorescence after infected by the scFv retrovirus. The cells were incubated with primary antibodies and the anti-mouse secondary antibody was CY3-conjugated. The images were captured by confocal laser scanning microscope (Carl Zeiss, ObErkochen, Germany). To avoid experimental error, non-infected U937 cells served as the control and the parameters of the microscope were fixed in each experiment. The typical field of the microscope was selected by the operator of the confocal laser scanning microscope.

\section{Programmed cell death (PCD) analysis}

The cells were stained with annexin-allophycocyanin (APC)/7amino-actinomycin (7-AAD) (BD PharMingen, San Jose, CA, USA) and evaluated using FCM. APC was optimized for FL-4 fluorescence on a flowcytometer, and 7-AAD fluorescence was detected in the far red range of the spectrum $(650 \mathrm{~nm}$ longpass filter $)^{[8]}$. The results were represented as the percentage of both annexin $\mathrm{V}-\mathrm{APC}^{+} / 7-\mathrm{AAD}^{-}$and annexin $\mathrm{V}-\mathrm{APC}^{+} / 7-\mathrm{AAD}^{+}$ cells. For confocal microscopy, the excitation wavelength of annexin V-APC was $633 \mathrm{~nm}$ and the emission wavelength was $700-780 \mathrm{~nm}^{[8]}$. The excitation wavelength of 7-AAD was 543 $\mathrm{nm}$ and the emission wavelength was 560-615 nm. A total of 200 cells were counted, and the results were calculated based on the percentages of both annexin $\mathrm{V}-\mathrm{APC}^{+} / 7-\mathrm{AAD}^{-}$and annexin $\mathrm{V}-\mathrm{APC}^{+} / 7-\mathrm{AAD}^{+}$cells.

Western blot was performed using PCD-associated antibodies. The Granzyme B, microtubule-associated protein 1 light chain 3 (LC-3) and Caspase 9 antibodies were purchased from Cell Signal Technology (MA, USA); AIF and Caspase 3 antibodies were purchased from Epitmics (CA, USA); antibodies against PARP were purchased from Santa Cruz Biotechnology (CA, USA); anti-Caspase 8 was purchased from BD Pharmingen (CA, USA).

\section{Changes in CHMP5 gene and protein expression after ScFv retrovirus infection}

The changes in gene and protein expression of CHMP5 in $\mathrm{U} 937$ cells after scFv retrovirus infection were studied. Reverse transcription polymerase chain reaction (RT-PCR) was performed, and the CHMP5 primers were: $5^{\prime}$-TGC TCA AGA TGA ACC GAC TCT-3' and 5'-GTG GGA ACA CCT TCT GGA AT-3'. The $\beta$-actin primers were: $5^{\prime}$-TCG ACA ACG GCT CCG GCA T- $3^{\prime}$ and $5^{\prime}$-AAG GTG TGG TGC CAG ATT TTC-3'. The polyclonal antibody against CHMP5 that was used for Western blot analysis was purchased from Abcam (Cambridge, UK). 


\section{Statistical analysis}

All statistics were performed using SAS 6.12 software. Data were expressed as mean $\pm S D$ from three independent experiments. The statistical significance was determined using $t$-test to compare groups of data. $P$ values $<0.05$ were considered to be statistically significant.

\section{Results}

Identification of anti-CHMP5 monoclonal antibody

Because a monoclonal antibody of CHMP5 was not commercially available and we needed to obtain the VL and $\mathrm{VH}$ sequence of anti-CHMP5 hybridoma cells, we constructed hybridoma cells and produced our own CHMP5 monoclonal antibody. After we obtained the monoclonal antibody of CHMP5, both Western blot analysis and immunofluorescence studies verified that McAb functioned as expected (Figure 1). The U937 group consisted of U937 cells that were not transfected with plasmids. The sh Con group consisted of U937 cells that were transfected with short-hairpin RNA (shRNA) control plasmids. The sh-CHMP5 group was composed of cells with RNA interference-mediated CHMP5 inhibition ${ }^{[8]}$. We found that our monoclonal antibody could detect an approximately $28 \mathrm{kDa}$ band in the U937 and sh Con groups by Western blot analysis; however, this band was very weak in the sh-CHMP5 group. The reduction in the density of the band was consistent with the changes in CHMP5 expression, indicating that this McAb could be utilized to detect CHMP5 protein by Western blot (Figure 1A). The McAb immunofluorescence (IF) results also confirmed that we had successfully prepared the McAb. Figure 1B-1D show the immunofluorescence results of the U937, sh Con and shCHMP5 groups, respectively. The figure shows the merged by red and blue fluorescence images. The red fluorescence (CY3) represents CHMP5 protein, and the blue fluorescence represents the DAPI (diamidino-phenyl-indole) signal, which labeles the nucleus. We found that the red fluorescence signal of the CHMP5 protein was highly expressed throughout cytosol (Figure 1A and 1B), but the red fluorescence signal decreased after CHMP5 was inhibited by RNA interference (Figure 1C). The change in red fluorescence was consistent with the changes in CHMP5 expression, indicating that this McAb may be utilized for immunofluorescence.

\section{VL and VH cloning}

The detailed DNA sequencing results of the cloned VL and $\mathrm{VH}$ of the scFv were supplied in the supplemental data.

IgBLAST and Nucleotide blast (http://blast.ncbi.nlm.nih. gov/Blast.cgi) were employed, and we confirmed that the sequences of $\mathrm{VL}$ and $\mathrm{VH}$ we obtained represented the variable region. VL shares $98 \%$ identity $(281 / 288)$ to the Mus musculus strain C57BL/6J chromosome 6 genomic contig (NT 039353.7). At this site, the Mus musculus light chain gene Igk-V21-12 is represented (GeneID: 667914). The VH was on the Mus musculus strain 129/SvJ chromosome 12 unlocalized genomic contig (NT 114985.2), and this site included the Mus musculus heavy chain gene Igh-VX24 (GeneID: 195176). The VH appeared to be the composite of two fragments, and each fragment showed high similarity (98\% and 100\%, respectively) to Igh-VX24.

\section{Changes in CHMP5 protein expression after ScFv retrovirus infection}

The non-infected U937 group showed CHMP5 protein expression (red fluorescence) distributed throughout the cytosol. The GFP protein could not be detected because it was not infected (Figure 2). The scFv-Con group (U937 cells were infected by empty pMIG-GFP) displayed GFP expression, indicating that the retrovirus had successfully infected U937 cells, and we could note that the expression of the CHMP5 protein was similar to the level observed in the U937 cells (Figure 2). The scFV-virus group (U937 cells infected with the pMIG-CHMP5 $\mathrm{scFv}$ retrovirus) showed GFP expression, indicating that the virus had successfully infected U937 cells. We found that the red fluorescence decreased dramatically, and the remaining red fluorescence appeared to exist as particulates in cytosol (Figure 2).

Changes in programmed cell death after ScFv retrovirus infection By FCM, we found that the apoptosis rate was $1.60 \% \pm 0.24 \%$ in U937 group, and $1.81 \% \pm 0.11 \%$ and $10.19 \% \pm 0.46 \%$ in the scFv-Con and scFv-CHMP5 groups, respectively. There were
A

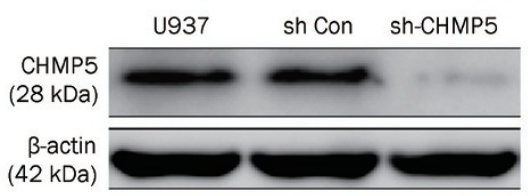

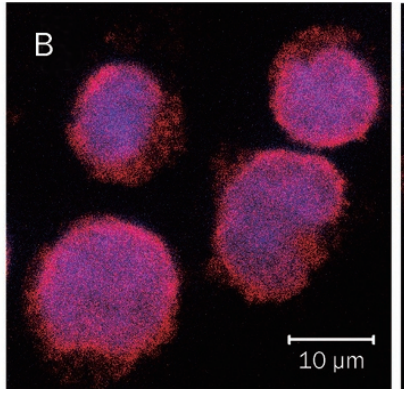
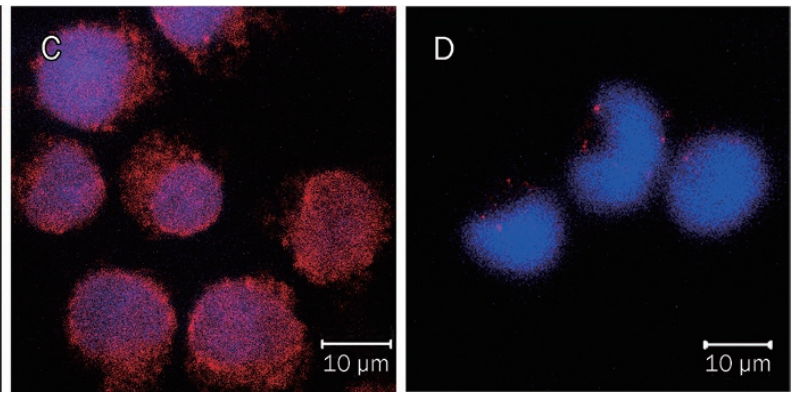

Figure 1. The efficiency of CHMP5 monoclonal antibody. (A) The CHMP5 McAb was able to detect the CHMP5 protein by Western blot. When $\mathrm{CHMP5}$ was inhibited by sh-CHMP5, the band was nearly undetectable; the McAb could detect the CHMP5 band in the control groups, in which CHMP5 was not inhibited. (B) In untranfected U937 cells, the red fluorescence signal of the CHMP5 protein was relatively highly expressed throughout cytosol. (C) In the sh Con group, the red fluorescence signal of the CHMP5 protein was similar to untranfected U937 cells. (D) The red fluorescence signal of CHMP5 protein decreased dramatically in the sh-CHMP5 group. 

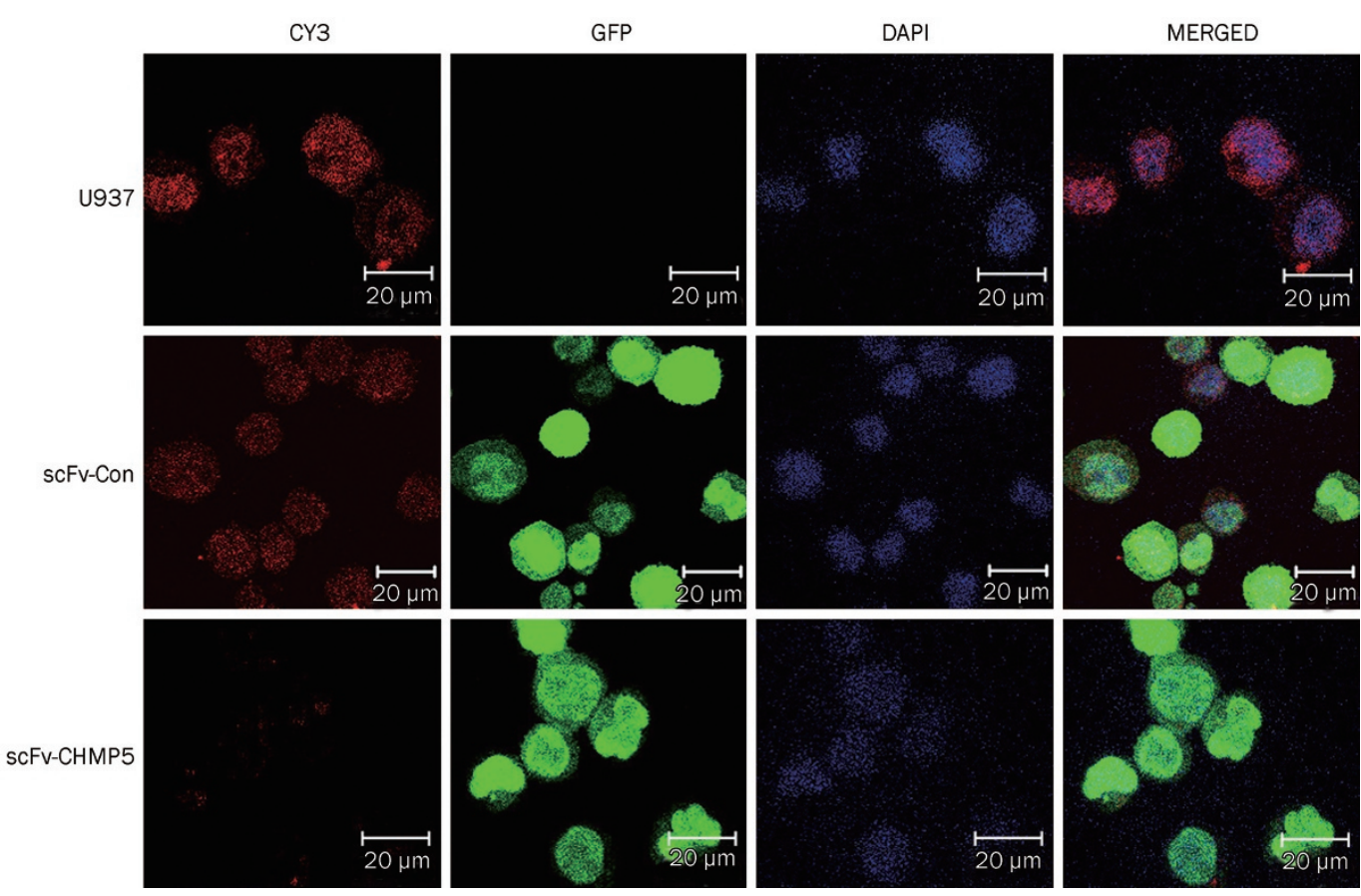

Figure 2. Immunofluorescence after scFv retrovirus infection. The U937 group consisted of cells not infected by the retrovirus, the scFv-Con group consisted of U937 cells infected by control retrovirus, and the scFv-CHMP5 group consisted of U937 cells infected by scFv retrovirus. CHMP5 was represented by the CY3 red fluorescence signal, the green fluorescence signal represented GFP, and DAPI was depicted by the blue fluorescence signal. The merged images represent the fusion of the red, green and blue fluorescence images. The expression of CHMP5 was similar in the U937 group and scFv-Con group; however, the signal of CHMP5 decreased dramatically in the ScFv-CHMP5 group.

no statistically significant differences between the U937 and scFv-Con groups $(P=0.1217)$. There was a statistically significant difference between the $\mathrm{scFv}$-Con and scFv-CHMP5 groups $(P=0.0015)$ (Figure $3 \mathrm{~A})$. The apoptosis rate increased approximately 5 times following the scFv retrovirus infection. We used confocal microscopy to further demonstrate that the apoptosis rate was $3.27 \%$ in the U937 group, $3.54 \%$ in the $\mathrm{scFv}$ Con group and $12.36 \%$ in the scFv-CHMP5 group, in agreement with the FCM results (Figure 3B-3D). We also found that the percentage of 7-AAD single-stained cells increased, which was likely necrosis ${ }^{[27,28]}$ (Figure 3D).
After we showed that the scFv retrovirus infection could induce U937 leukemic cells to undergo apoptosis, Western blot analysis was applied to study the changes in the apoptotic pathway. The autophagic and necrotic programmed cell death pathways were also examined. We found that after U937 cells were infected by the scFv retrovirus, the expression of AIF and cleaved Caspase 3 increased (Figure 4). PARP was the substrate of the activated Caspase 3, and we found that PARP decreased and its cleavage increased after scFv retrovirus infection. Granzyme B (Gran B), proCaspase 8, cleaved Caspase 8 and cleaved Caspase 9 showed no obvious changes.
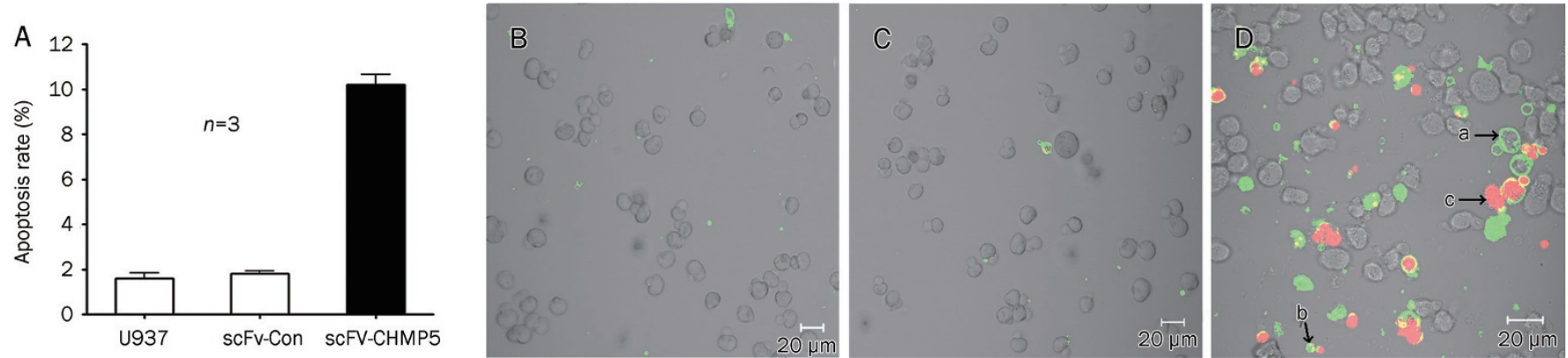

Figure 3. Apoptosis assessment after scFv retrovirus infection. (A) The apoptosis rate increased after infected by the scFv retrovirus and showed a statistically significant difference between the scFv-Con and scFv-CHMP5 groups, $P<0.05$ ( $n=3)$. (B) A typical confocal microscope field of the U937 group. (C) A typical confocal microscope field of the scFv-Con group. (D) A typical confocal microscope field of the scFv-CHMP5 group. (a) A typical annexin $\mathrm{V}-\mathrm{APC}^{+} / 7-\mathrm{AAD}^{-}$apoptotic cell at an early stage. (b) A typical annexin $\mathrm{V}-\mathrm{APC}^{+} / 7-\mathrm{AAD}^{+}$apoptotic cell at a later stage; we could view the karyorrhexis and apoptotic body. (c) Karyopyknosis, which is indicative of necrosis. 


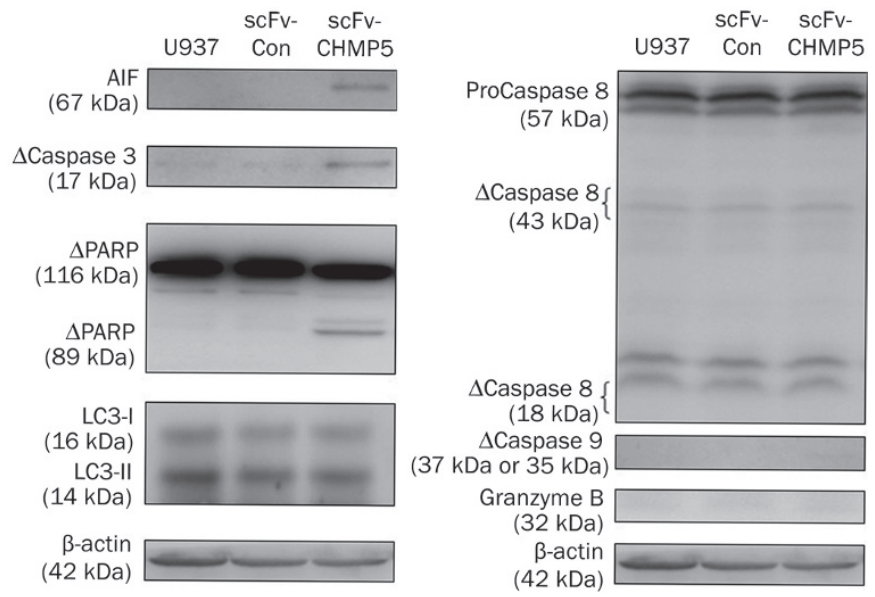

Figure 4. Expression of PCD-related factors detected by Western blot. After $U 937$ cells were infected by ScFv retrovirus, we found that the amount of AIF protein and cleaved Caspase 3 increased, and PARP, which was the substrate of activated Caspase 3, was cleaved. However, the levels of Granzyme B (Gran B), proCaspase 8, cleaved Caspase 8 and cleaved Caspase 9 were not changed. Neither the conversion of LC3-I to LC3-II nor an increase of LC3-II was observed. $\Delta$ indicated the cleaved protein.

Neither the conversion of LC3-I to LC3-II nor the increase of LC3-II was observed after $\mathrm{scFv}$ retrovirus infection (Figure 4).

\section{CHMP5 gene and protein changes after ScFv retrovirus infection}

To detect whether $\mathrm{scFv}$ retrovirus infection could affect the gene expression in U937 cells, reverse transcript PCR was used. We found the gene expression was not altered after scFv retrovirus infection. The change in $\mathrm{CHMP5}$ protein expression after $\mathrm{scFv}$ retrovirus infection was also studied by Western blot using a polyclonal antibody purchased from Abcam. We found that the protein expression was also not changed after $\mathrm{scFv}$ retrovirus infection (Figure 5).

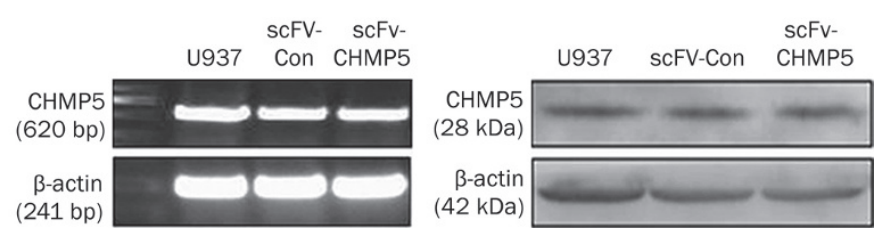

Figure 5. Changes in $\mathrm{CHMP5}$ gene and protein expression after ScFv retrovirus infection. (A) We did not observe gene expression changes. (B) We found that the protein expression was not changed after ScFv retrovirus infection.

\section{Discussion}

As we have mentioned previously, immunofluorescence experiments using a monoclonal antibody produced by our lab suggested that the CHMP5 protein was expressed at relatively low levels in the cytosol of 293T cells and normal blood mononuclear cells from healthy volunteers. Conversely, the
CHMP5 protein was highly expressed in the cytosol of AML leukemic cells (unpublished data). Moreover, our studies are consistent with previous studies which show that CHMP5 is an anti-apoptotic gene ${ }^{[8,9]}$. Furthermore, in agreement with these previous studies, we found that the CHMP5 gene expression is significantly increased in de novo or relapsed AML, indicating that it may participate in leukemogenesis ${ }^{[8,9]}$. As shown in Figure 1, after CHMP5 is inhibited by RNA interference in the AML leukemic cell line, U937, the expression of the CHMP5 protein is significantly decreased, and the leukemic cells are induced to undergo apoptosis ${ }^{[8]}$. These findings indicate that highly expressed CHMP5 protein in the cytosol may participate in leukemogenesis, and it may be a target for gene therapy.

The scFv to target molecules within cells provide a useful tool for research as well as for the control of diseases ${ }^{[13,15,16,21]}$. To produce a scFv-CHMP5, the hybridoma cells should be created, and subsequently the VL and $\mathrm{VH}$ variable region from the RNA of hybridoma cells must be obtained ${ }^{[29]}$. Immunofluorescence revealed that when the U937 cells were infected by the scFv-CHMP5 retrovirus, the red fluorescence of the CHMP5 protein decreased dramatically. Because the VL and $\mathrm{VH}$ variable regions of the CHMP5 $\mathrm{scFv}$ are generated from the RNA of hybridoma cells, which are also used to produce the McAb of CHMP5, the antigenic site of CHMP5 is shared and recognized by $\mathrm{scFv}$ and $\mathrm{McAb}$. Immunofluoresence with this monoclonal antibody shows a decrease of red fluorescence (CHMP5 protein) after scFv retrovirus infection. This reduction may be explained by two potential scenarios; after the CHMP5 protein is neutralized by the $\mathrm{scFv}$, the amount of CHMP5 protein in cytosol is not altered or CHMP5 is degraded. Both the gene and protein expression level of CHMP5 are studied, and the results indicate its gene expression level and the amount of CHMP5 protein in cytosol are not altered after being neutralized by scFv (Figure 5). Notably, we find little red fluorescence remains in the cytosol after $\mathrm{scFv}$-virus infection, and this red fluorescence appears to be in the particulates in the cytosol. Ward et al has reported that the CHMP5 protein is likely to be found in vesicles, which are thought to be lysosome and $\mathrm{MVB}^{[7]}$. Consistent with this observation, we suggest that the CHMP5 protein, which has been neutralized by $\mathrm{scFv}_{\mathrm{V}}$, is in cytosol, whereas the remaining CHMP5 protein is localized to the lysosome and MVB.

Recently, apoptosis was classified as type I programmed cell death ${ }^{[30]}$. Our previous study had found that both the Granzyme B/Perforin-mediated apoptotic and AIF-mediated necrotic programmed cell death pathways are activated during CHMP5 deficiencies, whereas the autophagic programmed cell death is not activated (unpublished data). Therefore, these PCD pathways were studied in U937 cells after ScFv retrovirus infection. In general, activation of Caspase 9 results in the initiation of the intrinsic pathway, whereas activation of Caspase 8 results in the initiation of the extrinsic pathway ${ }^{[31]}$. Activation of either pathway leads to activation of the downstream Caspase 3 and Caspase 7. We find that Caspase 3 is activated after $\mathrm{U} 937$ cells are infected by scFv retrovirus, indicating the 
apoptotic PCD pathway is activated. Neither Caspase 8 nor Caspase 9 is activated, indicating that neither intrinsic and extrinsic apoptotic PCD pathways are activated. To our surprise, the granzyme B/perforin-mediated Caspase-dependent apoptotic PCD pathway is also not activated. Although the apoptosis-inducing protein, AIF, increased after $\mathrm{scFv}$ retrovirus infection, AIF is thought to activiate Caspase-independent necrotic PCD pathways. That is, it is considered to be unable to active Caspase $3^{[32,33]}$. It appears that an additional uncommon Caspase-dependent apoptotic PCD pathway is activated, or this may indicate that AIF can active Caspase 3.

AIF-induced apoptosis now is reported to be Caspaseindependent necroptosis ${ }^{[32]}$, and necrosis is thought to be type III programmed cell death ${ }^{[30]}$. Increasing evidence shows that necrosis is a highly orchestrated type of $\mathrm{PCD}^{[32,33]}$. Upon activation of PCD, AIF is released from the mitochondria and translocated into the nucleus to induce phosphatidylserine exposure, chromatin condensation and DNA fragmentation ${ }^{[27,28]}$. We find that AIF increases after scFV retrovirus infection in U937 cells. Phosphatidylserine exposure can be detected by annexin $\mathrm{V}$, and we find that both phosphatidylserine exposure and chromatin condensation can be found in U937 cells after scFV retrovirus infection (Figure 3D), indicating that AIF-mediated caspase-independent necrosis is activated.

Recently, autophagy has also been shown to be a genetically programmed and regulated process by which cells undergo self-elimination. It is classified as type II programmed cell death $^{[30]}$. In mammals, the conversion of the microtubuleassociated protein 1 light chain 3 (LC3) from LC3-I (free form) to LC3-II (phosphatidylethanolamine-conjugated form) or the increase of LC3-II is a key step in autophagy ${ }^{[34]}$. We find that neither the conversion of protein LC3-I to LC3-II nor the amount of LC3-II is increased. These findings indicate that autophagic programmed cell death is not observed in U937 cells after scFV virus infection.

In conclusion, we find that the scFv-CHMP5 virus can neutralize the abnormally high levels of the CHMP5 protein in the cytosol of AML leukemic U937 cells, thereby inducing leukemic cells to undergo programmed cell death via AIF-mediated caspase-independent necrosis and apoptosis. Althought this is a pilot report, our findings provide a potential method to treat AML.

\section{Abbreviations}

AML, acute myeloid leukemia; CHMP5, charged multivesicular body protein 5; MVB, multivesicular body; ESCRT, endosomal sorting complex required for transport; $\mathrm{scFv}$, single chain variable fragments antibody; PCD, programmed cell death; AIF, apoptosis inducing factor.

\section{Acknowledgements}

This work was supported by the National Natural Science Foundation of China (30670881 and 81000212), the Shanghai Municipal Health Bureau (2009Y031) and a cooperative project of the Renji-Foundation Medical School (ZD0704). We would like to thank Dr Qiang-su GUO from the Key Laboratory for Reproductive Medicine, Shanghai Jiao Tong University School of Medicine, for his technical assistance.

\section{Author contribution}

Hai-rong WANG designed research, performed research and wrote the paper; Zhen-yu XIAO, Miao CHEN, Fei-long WANG, Jia LIU, Hua ZHONG, Ji-hua ZHONG, and Yanlin SHEN performed research and analyzed data; Ren-rong OU-YANG and Shu-ming PAN analyzed data and revised the paper.

\section{Supplementary information}

Supplementary tables are available at Acta Pharmacologica Sinica website of NPG.

\section{References}

1 Jabbour EJ, Estey E, Kantarjian HM. Adult acute myeloid leukemia. Mayo Clin Proc 2006; 81: 247-60.

2 Shim JH, Xiao C, Hayden MS, Lee KY, Trombetta ES, Pypaert M, et al. CHMP5 is essential for late endosome function and down-regulation of receptor signaling during mouse embryogenesis. J Cell Biol 2006; 172: 1045-56.

3 Katzmann DJ, Odorizzi G, Emr SD. Receptor downregulation and multivesicular-body sorting. Nat Rev Mol Cell Biol 2002; 3: 893-905.

4 Raiborg C, Rusten TE, Stenmark H. Protein sorting into multivesicular endosomes. Curr Opin Cell Biol 2003; 15: 446-55.

5 Raiborg C, Stenmark H. The ESCRT machinery in endosomal sorting of ubiquitylated membrane proteins. Nature 2009; 458: 445-52.

6 Wollert T, Hurley JH. Molecular mechanism of multivesicular body biogenesis by ESCRT complexes. Nature 2010; 464: 864-9.

7 Ward DM, Vaughn MB, Shiflett SL, White PL, Pollock AL, Hill J, et al. The role of LIP5 and CHMP5 in multivesicular body formation and HIV-1 budding in mammalian cells. J Biol Chem 2005 280: 1054855.

8 Wang HR, Gu CH, Zhu JY, Han JY, Zhong H, Chen FY, et al. PNAS-2: a novel gene probably participating in leukemogenesis. Oncology 2006; 71: 423-9.

9 Shahmoradgoli M, Mannherz O, Engel F, Heck S, Krämer A, Seiffert M, et al. Antiapoptotic function of charged multivesicular body protein 5: a potentially relevant gene in acute myeloid leukemia. Int J Cancer 2011; 128: 2865-71.

10 Accardi L, Di Bonito P. Antibodies in single-chain format against tumour-associated antigens: present and future applications. Curr Med Chem 2010; 17: 1730-55.

11 Huston JS, Levinson D, Mudgett-Hunter M, Tai MS, Novotný J, Margolies MN, et al. Protein engineering of antibody binding sites: recovery of specific activity in an anti-digoxin single-chain Fv analogue produced in Escherichia coli. Proc Natl Acad Sci U S A 1988; 85: 5879-83.

12 Kortt AA, Malby RL, Caldwell JB, Gruen LC, Ivancic N, Lawrence MC, et al. Recombinant anti-sialidase single-chain variable fragment antibody. Characterization, formation of dimer and higher-molecularmass multimers and the solution of the crystal structure of the singlechain variable fragment/sialidase complex. Eur J Biochem 1994; 221: 151-7.

13 Pérez-Martínez D, Tanaka T, Rabbitts TH. Intracellular antibodies and cancer: new technologies offer therapeutic opportunities. Bioessays 2010; 32: 589-98. 
14 Lu RM, Chang YL, Chen MS, Wu HC. Single chain anti-c-Met antibody conjugated nanoparticles for in vivo tumor-targeted imaging and drug delivery. Biomaterials 2011; 32: 3265-74.

15 Marasco WA, Haseltine WA, Chen SY. Design, intracellular expression, and activity of a human anti-human immunodeficiency virus type 1 gp120 single-chain antibody. Proc Natl Acad Sci U S A 1993; 90: 7889-93.

16 Levy-Mintz P, Duan L, Zhang H, Hu B, Dornadula G, Zhu M, et al. Intracellular expression of single-chain variable fragments to inhibit early stages of the viral life cycle by targeting human immunodeficiency virus type 1 integrase. J Virol 1996; 70: 8821-32.

17 Mabry R, Snavely M. Therapeutic bispecific antibodies: The selection of stable single-chain fragments to overcome engineering obstacles. IDrugs 2010; 13: 543-9.

18 ten Cate B, Bremer E, de Bruyn M, Bijma T, Samplonius D, Schwemmlein $M$, et al. A novel AML selective TRAIL fusion protein that is superior to Gemtuzumab Ozogamicin in terms of in vitro selectivity, activity and stability. Leukemia 2009; 23: 1389-97.

19 Olafsen T, Betting D, Kenanova VE, Salazar FB, Clarke P, Said J, et al. Recombinant anti-CD20 antibody fragments for small-animal PET imaging of B-cell lymphomas. J Nucl Med 2009; 50: 1500-8.

20 Geng SS, Feng J, Li Y, Sun Y, Gu X, Huang Y, et al. Binding activity difference of anti-CD20 scFv-Fc fusion protein derived from variable domain exchange. Cell Mol Immunol 2006; 3: 439-43.

21 Accardi L, Donà MG, Mileo AM, Paggi MG, Federico A, Torreri P, et al. Retinoblastoma-independent antiproliferative activity of novel intracellular antibodies against the E7 oncoprotein in HPV 16-positive cells. BMC Cancer 2011; 11: 17.

22 Ball RK, Siegl B, Quellhorst S, Brandner G, Braun DG. Monoclonal antibodies against simian virus 40 nuclear large $T$ tumour antigen: epitope mapping, papova virus cross-reaction and cell surface staining. EMBO J 1984; 3: 1485-91.

23 Lou Q, Kelleher RJ Jr, Sette A, Loyall J, Southwood S, Bankert RB, et al. Germ line tumor-associated immunoglobulin $\mathrm{VH}$ region peptides provoke a tumor-specific immune response without altering the response potential of normal B cells. Blood 2004; 104: 752-9.
24 Lu M, Gong XG, Yu H, Li JY. Cloning, expression, purification, and characterization of LC-1 ScFv with GFP tag. J Zhejiang Univ Sci B 2005; 6: 832-7.

25 Welm AL, Kim S, Welm BE, Bishop JM. MET and MYC cooperate in mammary tumorigenesis. Proc Natl Acad Sci U S A 2005; 102: 4324-9.

26 Wang L, O'Leary H, Fortney J, Gibson LF. $\mathrm{Ph}^{+} / \mathrm{VE}$-cadherin ${ }^{+}$identifies a stem cell like population of acute lymphoblastic leukemia sustained by bone marrow niche cells. Blood 2007; 110: 3334-44. .

27 Susin SA, Lorenzo HK, Zamzami N, Marzo I, Snow BE, Brothers GM, et al. Molecular characterization of mitochondrial apoptosis-inducing factor. Nature 1999; 397: 441-6.

28 Lee JH, Yang HS, Park KW, Kim JY, Lee MK, Jeong IY, et al. Mechanisms of thiosulfinates from Allium tuberosum L-induced apoptosis in HT-29 human colon cancer cells. Toxicol Lett 2009; 188: 142-7.

29 Colcher D, Pavlinkova G, Beresford G, Booth BJ, Batra SK. Singlechain antibodies in pancreatic cancer. Ann N Y Acad Sci 1999; 880: 263-80.

30 Portt L, Norman G, Clapp C, Greenwood M, Greenwood MT. Antiapoptosis and cell survival: a review. Biochim Biophys Acta 2011; 1813: 238-59. .

31 Klener P Jr, Andera L, Klener P, Necas E, Zivný J. Cell death signalling pathways in the pathogenesis and therapy of haematologic malignancies: Overview of apoptotic pathways. Folia Biol (Praha) 2006; 52: 34-44.

32 Delavallée L, Cabon L, Galán-Malo P, Lorenzo HK, Susin SA. AIFmediated caspase-independent necroptosis: a new chance for targeted therapeutics. IUBMB Life 2011; 63: 221-32.

33 Boujrad H, Gubkina O, Robert N, Krantic S, Susin SA. AlF-mediated programmed necrosis: a highly regulated way to die. Cell Cycle 2007; 6: 2612-9.

34 Kabeya Y, Mizushima N, Ueno T, Yamamoto A, Kirisako T, Noda T, et al. LC3, a mammalian homologue of yeast Apg8p, is localized in autophagosome membranes after processing. EMBO J 2000; 19: 5720-8. 